\title{
Ledipasvir/Sofosbuvir Effectively Treats Hepatitis C Virus Infections in an Underserved Population
}

\author{
Rachel A. Stewart ${ }^{1} \cdot$ Brooke R. MacDonald ${ }^{2} \cdot{\text { Tzu-Chun } \mathrm{Chu}^{2} \text {. Jonathan D. Moore }}^{3} \cdot$ Esther O. Fasanmi $^{4}$. \\ Rohit P. Ojha ${ }^{2,3}$
}

Received: 5 March 2018 / Accepted: 9 July 2018 / Published online: 16 July 2018

(c) The Author(s) 2018

\begin{abstract}
Background Underserved populations have an unequal burden of HCV infections and poor outcomes with interferon-based treatments. Direct-acting antivirals have the potential to reduce these inequalities.

Aims We aimed to estimate sustained virologic response (SVR) following treatment with sofosbuvir-based regimens for $\mathrm{HCV}$ infections among underserved individuals and summarize the frequency of SVR across published studies of underserved populations.

Methods We used data from a clinical cohort of patients aged $\geq 18$ years who initiated sofosbuvir-based regimens for HCV infection between February 2014 and June 2016 at an urban public hospital network that serves as the healthcare safety-net for Tarrant County, Texas. We estimated SVR with corresponding 95\% confidence limits (CL). In addition, we systematically reviewed the evidence to identify other studies of direct-acting antivirals among underserved populations.

Results Our study population comprised 435 patients. The majority of patients were aged $\geq 50$ years (76\%), male (52\%), non-Hispanic White (54\%), HCV genotype 1 (79\%) and treated with ledipasvir/sofosbuvir (69\%). Overall SVR was $89 \%$ (95\% CL 86, 92\%) and highest for ledipasvir/sofosbuvir (SVR=95\%, 95\% CL 92, 97\%). The reported SVR following directacting antivirals among 837 underserved patients from three other studies ranged between 90 and 99\%.

Conclusions Our results suggest that direct-acting antivirals, particularly ledipasvir/sofosbuvir, are generally effective for achieving SVR among underserved patients with HCV infections and may help reduce inequalities in HCV prevalence and outcomes for this vulnerable population.
\end{abstract}

Keywords Hepatitis C virus $\cdot$ Direct-acting antivirals $\cdot$ Underserved $\cdot$ Safety-net $\cdot$ Inequalities

Rohit P. Ojha

rojha@jpshealth.org

Rachel A. Stewart rstewa03@jpshealth.org

Brooke R. MacDonald bmacdona@jpshealth.org

Tzu-Chun Chu

tchu01@jpshealth.org

Jonathan D. Moore

jonathan.moore@my.unthsc.edu

Esther O. Fasanmi

efasanmi@jpshealth.org
1 Acclaim Gastroenterology, JPS Health Network, 1500 S Main St., Fort Worth, TX 76104, USA

2 Center for Outcomes Research, JPS Health Network, 1500 S Main St., Fort Worth, TX 76104, USA

3 Department of Biostatistics and Epidemiology, UNT Health Science Center School of Public Health, 3500 Camp Bowie Blvd., Fort Worth, TX 76107, USA

4 Pharmacy Services Administration, JPS Health Network, 1500 S Main St., Fort Worth, TX 76104, USA 


\section{Introduction}

The results of randomized controlled trials (RCTs) suggest that second-generation direct-acting antivirals have high efficacy and tolerability for achieving sustained virologic response (SVR) among individuals with hepatitis $\mathrm{C}$ virus (HCV) infections [1-5]. These findings supported the approval of sofosbuvir by the US Food and Drug Administration in 2013 and ledipasvir/sofosbuvir combination therapy in October 2014 [6]. The availability of efficacious and tolerable $\mathrm{HCV}$ treatment options fueled optimism about eradicating $\mathrm{HCV}$ infections and preventing progression to adverse outcomes such as cirrhosis and hepatocellular carcinoma. The results of RCTs are not always generalizable to target populations in real-world settings [7], but real-world studies of sofosbuvir-based regimens suggest SVR frequencies $>90 \%$ [8-13]. Nevertheless, the available real-world evidence is largely based on studies of patients with established access to care (e.g., integrated healthcare systems or insured populations) and may not be generalizable to the broader target population of HCVinfected individuals.

Underserved populations (e.g., racial/ethnic minorities, uninsured) have an unequal burden of HCV. For example, the prevalence of $\mathrm{HCV}$ is $\sim 7.0 \%$ among individuals at safety-net institutions (often the primary source of care for underserved populations) $[14,15]$, which is up to sevenfold higher than the general population [16]. Lack of eligibility for interferon-based regimens and poor response to treatment among the eligible exacerbated the inequalities in HCV outcomes for underserved populations [16-19]. The availability of direct-acting antivirals has made most underserved individuals eligible for HCV treatment [16], which could reduce inequalities, but limited evidence is available about the effectiveness among underserved individuals. This evidence would be particularly useful for informing real-world expectations of direct-acting antivirals among populations with barriers to care. Therefore, we aimed to estimate SVR following treatment with sofosbuvir-based regimens for HCV infections in a cohort of underserved individuals and summarize the frequency of SVR across published studies of underserved populations.

\section{Methods}

\section{Study Population}

We developed a clinical cohort based on electronic health records with prospectively documented treatment (baseline) and outcome (follow-up) [20, 21]. This cohort was derived from patients treated for HCV infection at the JPS Hepatology Clinic, which is part of the JPS Health Network, an urban public hospital network that serves as the healthcare safety-net for Tarrant County, Texas. Patients in the network are treated regardless of ability to pay. Patients eligible for our study were aged $\geq 18$ years and initiated interferon-free sofosbuvir-based regimens between February 2014 and June 2016. In addition, a negative screen for illicit drug use was required given payer restrictions. This study was approved by the North Texas Regional Institutional Review Board.

During the study period, the JPS Hepatology Clinic included 1.5 full-time equivalent (FTE) advanced practice providers (APP), 1.0 FTE nurse, and 0.20 FTE pharmacist. The APPs scheduled laboratory testing and imaging, and the nurse scheduled follow-up appointments. APPs monitored patients on treatment including assessment for compliance, on-treatment response, and adverse effects. For uninsured patients, an as-needed pharmacy technician facilitated completion of applications to patient assistance programs for $\mathrm{HCV}$ treatment through pharmaceutical companies. Liver transplantation was not an option for patients in the clinic, but eligible patients with insurance were referred to local institutions for transplants. Individuals with simple HCV/HIV coinfections were managed in the JPS Healing Wings Clinic, a specialized Ryan Whitefunded HIV clinic. HCV/HIV coinfections with decompensated cirrhosis and HCV/HBV coinfections were managed within the JPS Hepatology Clinic.

\section{Variables}

Our outcome of interest was SVR, which was defined as no detectable HCV RNA, measured by standard assays, in serum at 12 weeks post-treatment [22]. Sofosbuvir-based regimens in our population included ledipasvir/sofosbuvir, ledipasvir/sofosbuvir/ribavirin, sofosbuvir/ribavirin, and sofosbuvir/daclatasvir. The selection of treatment regimens was based on standard guidelines [23] and consideration of insurance restrictions. We also ascertained sociodemographic (age, sex, race/ethnicity), clinical [prior HCV treatment, HCV genotype, cirrhosis, HIV status, body mass index (BMI)], and lifestyle characteristics (based on self-reported history of substance use or history of ICD-9-CM diagnosis codes for substance abuse [24]) at the time HCV treatment was initiated. Cirrhosis was assessed by biopsy or imaging (ultrasound, computed tomography, or magnetic resonance). Decompensated cirrhosis was defined as documented history of ascites, hepatic encephalopathy, bleeding esophageal varices, or hepatocellular carcinoma. 


\section{Data Analysis}

We estimated overall and regimen-specific frequency of SVR. Patients with unknown SVR status because of treatment discontinuation were included in the analysis as not having achieved SVR. Nevertheless, we did not assume that the remaining patients who were lost to follow-up had SVR despite completing the duration of treatment because not taking the regimen as prescribed (i.e., secondary non-adherence [25]) could negatively affect SVR. In addition, complete-case analysis (i.e., exclusion of patients with missing SVR status from the analysis) could induce selection bias and increase variance [26-29]. Therefore, we used multiple imputation to predict SVR status for the remaining 80 patients without $\mathrm{HCV}$ viral load measured 12 weeks post-treatment completion. Multiple imputation is a commonly used approach that leverages information from observations (i.e., patients) without missing values to predict values for observations with missing values [27-29]. The logistic regression imputation model included age, gender, race/ethnicity, insurance status, alcohol use, cirrhosis, prior HCV treatment, illicit drug use, HIV status, HCV genotype, chronic kidney disease, and treatment regimen. These covariates were selected based on knowledge of potential factors associated with loss to follow-up in our population. The analyses were based on 40 imputations to enhance stability of SVR estimates for the study population.

\section{Systematic Review}

We searched PubMed/Medline to identify eligible studies through April 25, 2018, using the following search phrase: (HCV OR "hepatitis C") AND (direct-acting OR interferon-free) AND (safety-net OR underserved OR vulnerable). In addition, we manually reviewed reference lists of relevant articles (i.e., backtracing) to identify other potentially eligible studies. Potentially eligible studies were screened in duplicate to identify real-world studies of direct-acting antivirals conducted exclusively at safetynet institutions in the USA. We verified safety-net status using the National Association of Public Hospitals and Health Systems list [30]. Conference proceedings, editorials, and review articles were excluded. For eligible studies, we abstracted the proportion of individuals who achieved 12-week SVR, which was our primary measure of interest. To reduce potential selection bias, the denominator for this proportion was based on the number of patients who initiated treatment. In addition, we abstracted study-level information including sociodemographic characteristics and proportions with cirrhosis, substance abuse, nonadherence, and lost to follow-up. Given the small number of studies identified, we did not pursue a meta-analysis (Fig. 1).
Fig. 1 Selection of eligible studies based on a systematic review of the literature

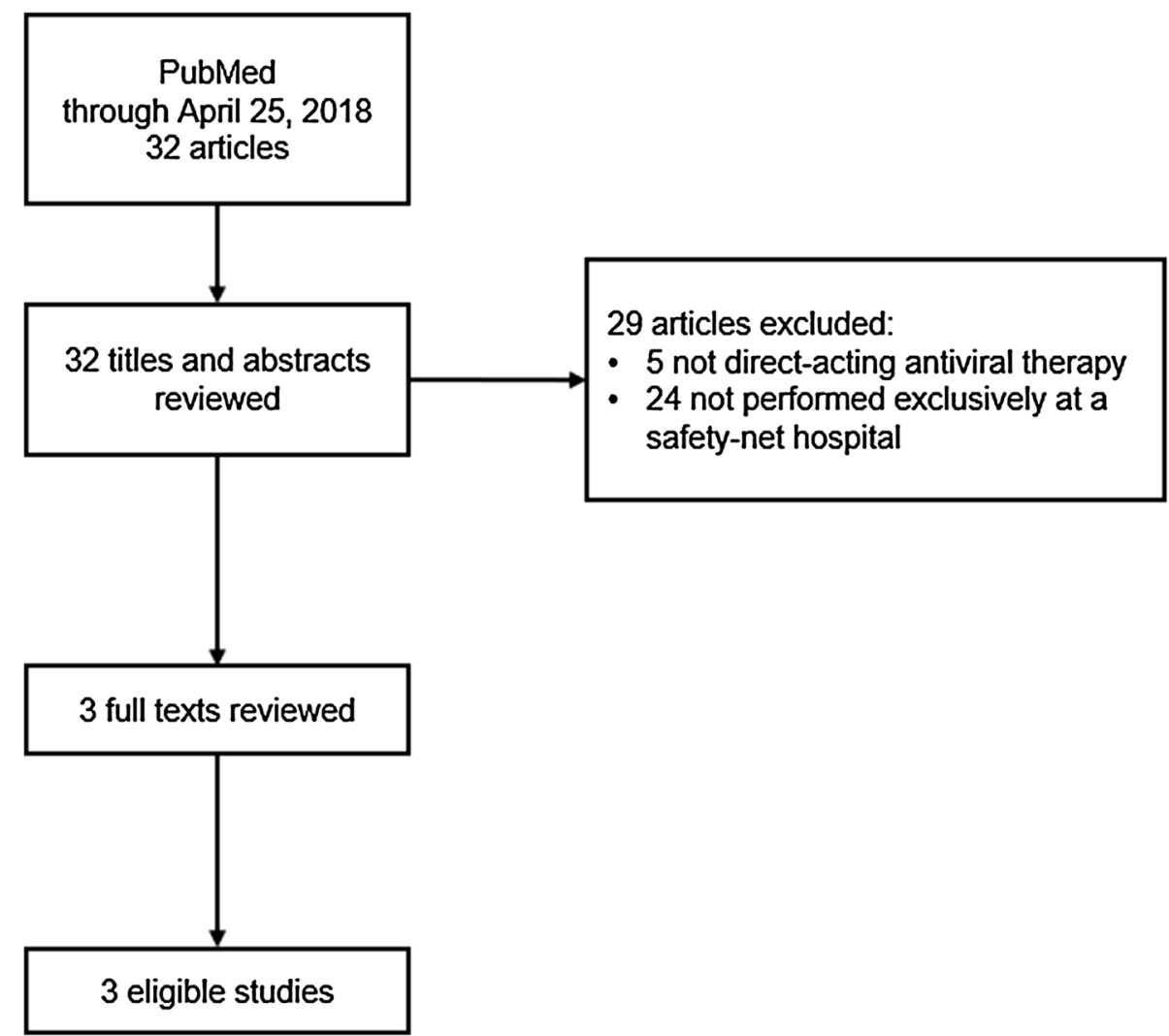




\section{Results}

We identified 448 patients with HCV infections aged $\geq 18$ years who initiated sofosbuvir-based regimens between January 2014 and June 2016. Small sample sizes for ledipasvir/sofosbuvir/ribavirin $(n=1)$ and sofosbuvir/daclatasvir $(n=12)$ precluded stable estimation of SVR frequency. These regimens were excluded from the analysis. Our study population thus comprised 435 patients. SVR status was unmeasured at 12 weeks postcompletion of treatment for $80(18 \%)$ patients, of whom 2 were unmeasured because of mortality during followup and 3 were non-adherent (i.e., early discontinuation). We observed 4 additional non-adherent patients, but these patients had SVR measured 12 weeks post-treatment completion (only 1 achieved SVR). Table 1 summarizes the characteristics of our study population by SVR status. The majority of patients in both groups were aged $\geq 50$ years, male, non-Hispanic White, overweight or obese, and HIVnegative. Most patients had no history of illicit drug use, but one-third of patients reported current alcohol use. HCV genotype 1 was most common among both groups. The prevalence of cirrhosis at treatment initiation was 25 and $16 \%$ among patients with known and unknown SVR status, respectively, of whom $18 \%$ overall had decompensated cirrhosis. Prior HCV treatment was documented for $17 \%$ of patients with known SVR status and 9.2\% with unknown SVR status. Most patients were treated with ledipasvir/sofosbuvir. In particular, 38\% were treated with 8-week ledipasvir/sofosbuvir, which is contraindicated for patients with cirrhosis.

Table 2 summarizes the proportions of patients who achieved SVR. Sofosbuvir-based regimens overall had SVR of $89 \%$ (95\% CL $86,92 \%$ ) with limited variation between subgroups except for gender. SVR for patients who initiated ledipasvir/sofosbuvir regimens was $95 \%$ (95\% CL 92, 97\%) with limited variation by subgroups. Overall SVR for patients treated with sofosbuvir/ribavirin was $76 \%$ (95\% CL 69, 84\%), and particularly low for males (SVR $=63 \%, 95 \%$ CL 50, 75\%), non-Hispanic Blacks (SVR $=53 \%, 95 \%$ CL 30, 75\%), HCV genotype 1 (SVR $=54 \%, 95 \%: 38,70 \%)$, patients with cirrhosis (SVR $=59 \%, 95 \%$ CL 40, 78\%), and treatment-experienced (SVR $=57 \%, 95 \%$ CL 35, 79\%).

Table 3 summarizes the characteristics of studies that assessed the real-world effectiveness of direct-acting antivirals among underserved populations. Our search identified 26 potentially eligible studies, of which three studies were eligible after full-text review, and we included the current study. The sample sizes for the four studies ranged from 121 to 523 patients, for a total of 1360 patients across studies. The median age was $\geq 50$ years in three out of
Table 1 Characteristics of patients who initiated sofosbuvir regimens for hepatitis C virus (HCV) infection between January 2014 and June 2016 by known or unknown 12 -week sustained virologic response (SVR) status

\begin{tabular}{|c|c|c|}
\hline Characteristic & $\begin{array}{l}\text { Known SVR } \\
n(\%)\end{array}$ & $\begin{array}{l}\text { Unknown SVR } \\
n(\%)\end{array}$ \\
\hline Overall & 355 & 80 \\
\hline \multicolumn{3}{|l|}{ Age category } \\
\hline$<50$ years & $71(20)$ & $34(42)$ \\
\hline$\geq 50$ years & $284(80)$ & $46(58)$ \\
\hline \multicolumn{3}{|l|}{ Gender } \\
\hline Male & $183(52)$ & $39(49)$ \\
\hline Female & $172(48)$ & $41(51)$ \\
\hline \multicolumn{3}{|l|}{ Racelethnicity } \\
\hline Non-Hispanic White & $201(57)$ & $50(63)$ \\
\hline Non-Hispanic Black & $107(30)$ & $15(19)$ \\
\hline Hispanic & $30(8.5)$ & $10(13)$ \\
\hline Other $^{\mathrm{a}}$ & $17(4.8)$ & $5(6.3)$ \\
\hline \multicolumn{3}{|l|}{ Insurance type } \\
\hline Uninsured & 274 (77) & $72(90)$ \\
\hline Medicare or medicaid & $51(14)$ & $5(6.3)$ \\
\hline Private & $30(8.5)$ & $3(3.8)$ \\
\hline \multicolumn{3}{|l|}{ Current alcohol use $^{\mathrm{b}}$} \\
\hline Yes & $103(30)$ & $23(30)$ \\
\hline No & $240(70)$ & $54(70)$ \\
\hline \multicolumn{3}{|c|}{ History of illicit drug use $\mathrm{b}^{\mathrm{b}}$} \\
\hline Yes & $53(16)$ & $10(13)$ \\
\hline No & $286(84)$ & $67(87)$ \\
\hline \multicolumn{3}{|l|}{ Body mass index } \\
\hline Obese $(\geq 30)$ & $153(43)$ & $26(33)$ \\
\hline Overweight (25-29.9) & $113(32)$ & $30(38)$ \\
\hline Normal (18.5-24.9) & $81(23)$ & $24(30)$ \\
\hline Underweight $(<18.5)$ & $8(2.3)$ & $0(0)$ \\
\hline \multicolumn{3}{|l|}{ HCV genotype } \\
\hline 1 & $275(77)$ & $62(78)$ \\
\hline 2 & $43(12)$ & $7(8.8)$ \\
\hline 3 & $33(9.3)$ & $9(11)$ \\
\hline 4 or 6 & $4(1.1)$ & $2(2.5)$ \\
\hline \multicolumn{3}{|l|}{$H I V$} \\
\hline Yes & $17(4.8)$ & $0(0)$ \\
\hline No & $338(95)$ & $80(96)$ \\
\hline \multicolumn{3}{|l|}{ Cirrhosis } \\
\hline Yes & $87(25)$ & $13(16)$ \\
\hline No & $268(75)$ & $67(84)$ \\
\hline \multicolumn{3}{|c|}{ Decompensated cirrhosis ${ }^{\mathrm{b}}$} \\
\hline Yes & $16(19)$ & $2(14)$ \\
\hline No & $70(81)$ & $12(86)$ \\
\hline \multicolumn{3}{|l|}{ Prior $H C V$ treatment $^{\mathrm{c}}$} \\
\hline Yes & $52(15)$ & $9(11)$ \\
\hline No & $302(85)$ & $71(89)$ \\
\hline \multicolumn{3}{|l|}{ Treatment regimen } \\
\hline Ledipasvir/sofosbuvir & $239(67)$ & $62(78)$ \\
\hline Ribavirin/sofosbuvir & $116(33)$ & $18(22)$ \\
\hline
\end{tabular}

${ }^{\mathrm{a}}$ Includes Asian and mixed ethnicity

${ }^{\mathrm{b}}$ Among individuals with cirrhosis

${ }^{\mathrm{c}}$ Missing values for some patients 
Table 2 Overall and subgroupspecific frequency of sustained virologic response following direct-acting antivirals among patients with hepatitis $\mathrm{C}$ virus infection at an urban safety-net institution

\begin{tabular}{|c|c|c|c|c|c|c|}
\hline & $n$ & $\begin{array}{l}\text { Overall } \\
\text { SVR (95\% CL) }\end{array}$ & $n$ & $\begin{array}{l}\text { Ledipasvir/sofosbuvir } \\
\text { SVR (95\% CL) }\end{array}$ & $n$ & $\begin{array}{l}\text { Sofosbuvir/ribavirin } \\
\text { SVR }(95 \% \mathrm{CL})\end{array}$ \\
\hline Overall & 435 & $89 \%(86 \%, 92 \%)$ & 301 & $95 \%(92 \%, 97 \%)$ & 134 & $76 \%(69 \%, 84 \%)$ \\
\hline \multicolumn{7}{|l|}{ Age } \\
\hline$<50$ years & 105 & $92 \%(85 \%, 98 \%)$ & 74 & $94 \%(87 \%, 100 \%)$ & 31 & $87 \%(73 \%, 100 \%)$ \\
\hline$\geq 50$ years & 330 & $88 \%(84 \%, 92 \%)$ & 227 & $95 \%(92 \%, 98 \%)$ & 103 & $73 \%(64 \%, 82 \%)$ \\
\hline \multicolumn{7}{|l|}{ Gender } \\
\hline Male & 213 & $82 \%(77 \%, 88 \%)$ & 158 & $90 \%(85 \%, 95 \%)$ & 64 & $63 \%(50 \%, 75 \%)$ \\
\hline Female & 222 & $96 \%(93 \%, 99 \%)$ & 143 & $99 \%(97 \%, 100 \%)$ & 70 & $89 \%(80 \%, 97 \%)$ \\
\hline \multicolumn{7}{|l|}{ Racelethnicity } \\
\hline Non-Hispanic White & 251 & $91 \%(87 \%, 94 \%)$ & 157 & $96 \%(92 \%, 99 \%)$ & 94 & $82 \%(74 \%, 91 \%)$ \\
\hline Non-Hispanic Black & 122 & $84 \%(77 \%, 91 \%)$ & 99 & $92 \%(86 \%, 98 \%)$ & 23 & $53 \%(30 \%, 75 \%)$ \\
\hline Hispanic & 40 & $89 \%(79 \%, 99 \%)$ & 30 & $96 \%(88 \%, 100 \%)$ & 10 & $69 \%(38 \%, 100 \%)$ \\
\hline Other & 22 & $94 \%(83 \%, 100 \%)$ & 15 & $98 \%(88 \%, 100 \%)$ & 7 & $86 \%(57 \%, 100 \%)$ \\
\hline \multicolumn{7}{|l|}{ HCV genotype } \\
\hline 1 & 337 & $89 \%(86 \%, 93 \%)$ & 295 & $94 \%(91 \%, 97 \%)$ & 42 & $54 \%(38 \%, 70 \%)$ \\
\hline 2 & 50 & $90 \%(82 \%, 99 \%)$ & 0 & a & 50 & $90 \%(82 \%, 99 \%)$ \\
\hline 3 & 42 & $81 \%(69 \%, 94 \%)$ & 0 & a & 42 & $81 \%(69 \%, 94 \%)$ \\
\hline \multicolumn{7}{|l|}{ Cirrhosis } \\
\hline Yes & 100 & $84 \%(76 \%, 91 \%)$ & 72 & $93 \%(86 \%, 99 \%)$ & 28 & $59 \%(40 \%, 78 \%)$ \\
\hline No & 335 & $91 \%(87 \%, 94 \%)$ & 229 & $95 \%(92 \%, 98 \%)$ & 106 & $81 \%(73 \%, 89 \%)$ \\
\hline \multicolumn{7}{|l|}{ Prior HCV treatment } \\
\hline Yes & 61 & $84 \%(74 \%, 93 \%)$ & 38 & $99 \%(97 \%, 100 \%)$ & 23 & $57 \%(35 \%, 79 \%)$ \\
\hline No & 374 & $90 \%(87 \%, 93 \%)$ & 263 & $94 \%(91 \%, 97 \%)$ & 111 & $81 \%(73 \%, 89 \%)$ \\
\hline
\end{tabular}

four of the studies. The majority of the patients were nonHispanic Black in one study [31], whereas the majority of the patients in the remaining studies were non-Hispanic White. The frequency of uninsured patients ranged from 5 [32] to $79 \%$. The frequency of patients with a history of substance abuse was $\geq 50 \%$ in two studies $[31,32]$. The current study had the lowest frequency of patients with cirrhosis (22\%). Ledipasvir/sofosbuvir was the most common regimen across studies.

\section{Discussion}

Underserved patients had poor outcomes with interferonbased regimens and are often labeled "difficult to treat." Our study population comprised largely uninsured patients with substantial barriers to care who were dependent on patient assistance programs for treatment with direct-acting antivirals. The results of our study suggest that sofosbuvir-based regimens are generally effective for achieving SVR among underserved patients with $\mathrm{HCV}$ infections. Ledipasvir/sofosbuvir is particularly effective overall and within sociodemographic or clinical subgroups of the population.
To provide context for our findings, we systematically reviewed published literature to identify reports of SVR after direct-acting antivirals at safety-net institutions, where underserved populations primarily receive care. Prior studies [31-33] excluded patients with missing SVR status 12 weeks after completion of treatment. Such exclusions, inappropriately referred to as "intention to treat" but better known as complete-case analysis, can induce selection bias and overestimate SVR [26-29, 34]. This overestimation may be particularly severe for studies where loss to follow-up is high [35]. Biased SVR estimates from inappropriate exclusion in prior studies are also a concern in real-world studies of direct-acting antivirals among patients with established access to care [8-13]. Nevertheless, our estimate of 95\% SVR for patients treated with ledipasvir/sofosbuvir is similar to patients with established access to care. For example, Butt et al. [9] reported 96\% SVR for patients in the ERCHIVES cohort and Lai et al. [13] reported 94\% SVR for patients in the Kaiser Permanente-integrated health system. The negligible differences in response to treatment between diverse populations warrant further scrutiny of insurance restrictions on eligibility for ledipasvir/sofosbuvir.

Certain limitations should be considered when interpreting our results. In particular, the choice of any analytic 


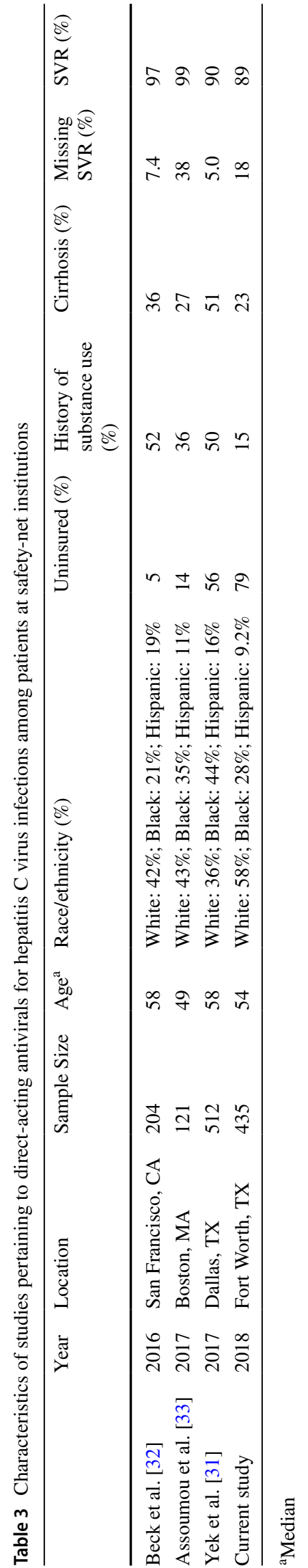

approach requires acceptance of a trade-off. We addressed missing information about SVR using multiple imputation to reduce potential selection bias from excluding these patients, which has been a limitation in prior studies. The trade-off is potential misclassification of SVR status if the imputed value was incorrect. Multiple imputation may not be superior to other approaches in certain situations [29, 36]. Unfortunately, an assessment of which approach would result in less bias would require knowing the true SVR status for patients who were lost to follow-up, which is not always possible among underserved populations because of challenges with follow-up. Nevertheless, future studies that aim to estimate SVR in real-world settings should include all patients who initiated therapy as part of the analysis [34]. Non-adherence to therapy and loss to follow-up during therapy are key reasons for not achieving SVR [37]. The exclusion of patients from the analysis because of nonadherence or missing SVR status because of loss to followup is also inappropriate [34]. A more informative approach is to explore reasons for non-adherence or loss to follow-up, which could be targeted by interventions to enhance adherence or retention, respectively. Patient navigation programs may be a consideration to improve adherence and reduce loss to follow-up. Such programs have been successful in achieving a high frequency of SVR [38].

In summary, underserved populations, which include racial/ethnic minorities and the uninsured, have the highest burden of HCV but low SVR following interferon-based treatments [14, 15, 17, 39]. Our findings suggest that SVR among underserved populations, whether in our setting or other settings, are similar to populations with established access to care. Our findings may help redefine providers' expectations about SVR when treating underserved individuals. Our study also provides encouraging evidence for the potential reduction of persistent inequalities in $\mathrm{HCV}$ prevalence and poor outcomes for this population. Nevertheless, the assessment of SVR at 12 weeks post-completion of treatment alone provides limited understanding about longterm outcomes in underserved populations because of the potential for reinfection among people who inject drugs. The assessment of long-term outcomes such as cirrhosis and hepatocellular carcinoma is necessary for more comprehensive understanding about treatment effects. Lastly, patientreported outcomes should be assessed in future studies of direct-acting antiviral regimens to enhance shared decisionmaking based on potential trade-offs between regimen effectiveness, side effects, and patient perceptions [40].

Acknowledgments The authors are grateful to Sajid Shaikh for assistance with data collection.

Author's contribution RAS contributed to study design, data collection and drafted manuscript. BRM and TC involved in data collection, data analysis, critical review of manuscript draft. JDM helped with 
data analysis, critical review of manuscript draft. EOF contributed to data collection, critical review of manuscript draft. RPO involved in study design, data analysis and drafted manuscript. The final version of this manuscript, including the authorship list, has been approved by all authors.

\section{Compliance with ethical standards}

Conflict of interest The authors declare that they have no conflict of interest.

Open Access This article is distributed under the terms of the Creative Commons Attribution-NonCommercial 4.0 International License (http://creativecommons.org/licenses/by-nc/4.0/), which permits any noncommercial use, distribution, and reproduction in any medium, provided you give appropriate credit to the original author(s) and the source, provide a link to the Creative Commons license, and indicate if changes were made.

\section{References}

1. Curry MP, Tapper EB, Bacon B, et al. Effectiveness of 8- or 12-weeks of ledipasvir and sofosbuvir in real-world treatmentnaive, genotype 1 hepatitis $\mathrm{C}$ infected patients. Aliment Pharmacol Ther. 2017;46:540-548.

2. Kowdley KV, Gordon SC, Reddy KR, et al. Ledipasvir and sofosbuvir for 8 or 12 weeks for chronic HCV without cirrhosis. $N$ Engl J Med. 2014;370:1879-1888.

3. Terrault NA, Zeuzem S, Di Bisceglie AM, et al. Effectiveness of ledipasvir-sofosbuvir combination in patients with hepatitis $\mathrm{C}$ virus infection and factors associated with sustained virologic response. Gastroenterology. 2016;151:1131-1140. (e1135).

4. Andreone P, Colombo MG, Enejosa JV, et al. ABT-450, ritonavir, ombitasvir, and dasabuvir achieves $97 \%$ and $100 \%$ sustained virologic response with or without ribavirin in treatment-experienced patients with HCV genotype 1b infection. Gastroenterology. 2014;147:359-365. (e351).

5. Poordad F, Sievert W, Mollison L, et al. Fixed-dose combination therapy with daclatasvir, asunaprevir, and beclabuvir for noncirrhotic patients with HCV genotype 1 infection. Jama. 2015;313:1728-1735.

6. Qian XJ, Zhu YZ, Zhao P, Qi ZT. Entry inhibitors: new advances in HCV treatment. Emerg Microbes Infect. 2016;5:e3.

7. Rothwell PM. Commentary: external validity of results of randomized trials: disentangling a complex concept. Int J Epidemiol. 2010;39:94-96.

8. Backus LI, Belperio PS, Shahoumian TA, Loomis TP, Mole LA. Real-world effectiveness of ledipasvir/sofosbuvir in 4,365 treatment-naive, genotype 1 hepatitis $\mathrm{C}$-infected patients. Hepatology (Baltimore, Md.). 2016;64:405-414.

9. Butt AA, Yan P, Marks K, Shaikh OS, Sherman KE. Adding ribavirin to newer DAA regimens does not affect SVR rates in $\mathrm{HCV}$ genotype 1 infected persons: results from ERCHIVES. Aliment Pharmacol Ther. 2016;44:728-737.

10. Ioannou GN, Beste LA, Chang MF, et al. Effectiveness of sofosbuvir, ledipasvir/sofosbuvir, or paritaprevir/ritonavir/ombitasvir and dasabuvir regimens for treatment of patients with hepatitis $\mathrm{C}$ in the Veterans Affairs National Health Care System. Gastroenterology. 2016;151:457-471. (e455).

11. Sulkowski MS, Vargas HE, Di Bisceglie AM, et al. Effectiveness of simeprevir plus sofosbuvir, with or without ribavirin, in real-world patients with HCV genotype 1 infection. Gastroenterology. 2016;150:419-429.

12. Barron J, Xie Y, Wu SJ, et al. Treatment of chronic hepatitis $\mathrm{C}$ infection with sofosbuvir-based regimens in a commercially insured patient population. Am Health Drug Benefits. 2016;9:327-335.

13. Lai JB, Witt MA, Pauly MP, et al. Eight- or 12-week treatment of hepatitis $\mathrm{C}$ with ledipasvir/sofosbuvir: real-world experience in a large integrated health system. Drugs. 2017;77:313-318.

14. Coyle C, Kwakwa H, Viner K. Integrating routine HCV testing in primary care: lessons learned from five federally qualified health centers in Philadelphia, Pennsylvania, 2012-2014. JMIR Public Health Surveill. 2016;131:65-73.

15. Turner BJ, Taylor BS, Hanson J, et al. High priority for hepatitis C screening in safety net hospitals: results from a prospective cohort of 4582 hospitalized baby boomers. Hepatology (Baltimore, Md.). 2015;62:1388-1395.

16. Stepanova M, Younossi ZM. Interferon-free regimens for Chronic hepatitis c: barriers due to treatment candidacy and insurance coverage. Dig Dis Sci. 2015;60:3248-3251. https://doi.org/10.1007/ s10620-015-3709-6.

17. Nordstrom EM, Keniston A, Baouchi F, Martinez-Camacho A. Interferon-based hepatitis $\mathrm{C}$ therapy in a safety net hospital: access, efficacy, and safety. Eur J Gastroenterol Hepatol. 2017;29:10-16.

18. Schaeffer S, Khalili M. Reasons for HCV non-treatment in underserved African Americans: implications for treatment with new therapeutics. Ann Hepatol. 2015;14:234-242.

19. Donepudi I, Paredes A, Hubbard S, Awad C, Sterling RK. Utility of evaluating HCV in an uninsured population. Dig Dis Sci. 2015;60:1092-1097. https://doi.org/10.1007/s10620-014-3416-8.

20. Mathes T, Pieper D. Study design classification of registry-based studies in systematic reviews. J Clin Epidemiol. 2018;93:84-87.

21. Casey JA, Schwartz BS, Stewart WF, Adler NE. Using electronic health records for population health research: a review of methods and applications. Annu Rev Public Health. 2016;37:61-81.

22. Yoshida EM, Sulkowski MS, Gane EJ, et al. Concordance of sustained virological response 4,12 , and 24 weeks post-treatment with sofosbuvir-containing regimens for hepatitis C virus. Hepatology. 2015;61:41-45.

23. AASLD/IDSA HCV Guidance Panel. Hepatitis C guidance: AASLD-IDSA recommendations for testing, managing, and treating adults infected with hepatitis C virus. Hepatology. 2015;62:932-954.

24. Healthcare Cost and Utilization Project (HCUP) Statistical Briefs [Internet]. Rockville, MD: Agency for Healthcare Research and Quality (US). 2006. Available from https://www.ncbi.nlm.nih. gov/books/NBK52651/.

25. Solomon MD, Majumdar SR. Primary non-adherence of medications: lifting the veil on prescription-filling behaviors. J Gen Intern Med. 2010;25:280-281.

26. Greenland S, Finkle WD. A critical look at methods for handling missing covariates in epidemiologic regression analyses. Am J Epidemiol. 1995;142:1255-1264.

27. Donders AR, van der Heijden GJ, Stijnen T, Moons KG. Review: a gentle introduction to imputation of missing values. J Clin Epidemiol. 2006;59:1087-1091.

28. Groenwold RH, Donders AR, Roes KC, Harrell FE Jr, Moons KG. Dealing with missing outcome data in randomized trials and observational studies. Am J Epidemiol. 2012;175:210-217.

29. Sterne JA, White IR, Carlin JB, et al. Multiple imputation for missing data in epidemiological and clinical research: potential and pitfalls. BMJ. 2009;338:b2393.

30. Zaman OS, Cummings LC, Laycox S. America's safety net hospitals and health systems, 2010: results of the annual NAPH 
hospital characteristics survey. National Association of Public Hospitals and Health Systems; 2012.

31. Yek C, de la Flor C, Marshall J, et al. Effectiveness of direct-acting antiviral therapy for hepatitis $\mathrm{C}$ in difficult-to-treat patients in a safety-net health system: a retrospective cohort study. BMC Med. 2017; 15:204

32. Beck KR, Kim N, Khalili M. Sofosbuvir-containing regimens for chronic hepatitis $\mathrm{C}$ are successful in the safety-net population: a real-world experience. Dig Dis Sci. 2016;61:3602-3608. https:// doi.org/10.1007/s10620-016-4340-x.

33. Assoumou SA, Huang W, Young K, Horsburgh CR, Linas BP. Real-world outcomes of hepatitis $\mathrm{C}$ treatment during the interferon-free era at an Urban Safety-net Hospital. J Health Care Poor Undeserved. 2017;28:1333-1344.

34. Ojha RP, Steyerberg EW. Real-world data on antiviral treatments for hepatitis C virus infections: can we define intention to treat or per protocol analyses? J Hepatol. 2018. https://doi.org/10.1016/j. jhep.2018.02.037.

35. Hernan MA, Hernandez-Diaz S, Robins JM. A structural approach to selection bias. Epidemiology. 2004;15:615-625.
36. Lewin A, Brondeel R, Benmarhnia T, Thomas F, Chaix B. Attrition bias related to missing outcome data: a longitudinal simulation study. Epidemiology. 2018;29:87-95.

37. Kattakuzhy S, Gross C, Emmanuel B, et al. Expansion of treatment for hepatitis $\mathrm{C}$ virus infection by task shifting to communitybased nonspecialist providers: a nonrandomized clinical trial. Ann Intern Med. 2017;167:311-318.

38. Ford MM, Johnson N, Desai P, Rude E, Laraque F. From care to cure: demonstrating a model of clinical patient navigation for hepatitis $\mathrm{C}$ care and treatment in high-need patients. Clin Infect Dis. 2017;64:685-691.

39. Mir HM, Stepanova M, Afendy M, Kugelmas M, Younossi ZM. African americans are less likely to have clearance of hepatitis $\mathrm{C}$ virus infection: the findings from recent U.S. population data. $J$ Clin Gastroenterol. 2012;46:e62-e65.

40. Younossi Z, Henry L. Systematic review: patient-reported outcomes in chronic hepatitis $\mathrm{C}$ - the impact of liver disease and new treatment regimens. Aliment Pharmacol Ther. 2015;41:497-520. 